\title{
STICKS study - Short-sTretch Inelastic Compression bandage in Knee Swelling following total knee arthroplasty - a feasibility study
}

T. M. Brock ${ }^{1 *}$, A. P. Sprowson ${ }^{\wedge}$, S. Muller ${ }^{3}$ and M. R. Reed ${ }^{3}$

\begin{abstract}
Background: Postoperative knee swelling is common and impairs early postoperative function following total knee arthroplasty. It was hypothesised that the use of a short-stretch, inelastic compression bandage would reduce knee swelling and improve pain and early function. The aim of this study was to provide preliminary data and test feasibility with a view to informing a larger, future trial.

Methods: Fifty consecutive patients selected for primary total knee arthroplasty underwent distance randomisation to receive a short-stretch, inelastic compression bandage or a standard wool and crepe bandage for the first $24 \mathrm{~h}$ postoperatively. Study feasibility including recruitment rates, retention rates and complications were analysed. The Oxford Knee Score, the EQ-5D-3L index score, knee swelling, knee range of motion, visual analogue pain score and length of stay were compared between groups. Analysis of covariance (ANCOVA) was performed adjusting for the preoperative measurement.

Results: Sixty-eight percent of eligible patients were recruited into the trial. The retention rate was $88 \%$. There were no complications regarding compression bandage use. There was a greater mean but non-significant improvement in Oxford Knee Score ( $p=0.580$; point estimate $=2.1 ; 95 \% \mathrm{Cl}-3.288$ to 7.449$)$ and EQ-5D-3L index score $(p=0.057$; point estimate $=0.147 ; 95 \% \mathrm{Cl}-0.328$ to 0.005$)$ in the compression bandage group at 6 months. There was no significant difference between groups regarding knee swelling, knee range of motion, visual analogue pain score, complications and length of stay.
\end{abstract}

Conclusion: Preliminary data suggests that the use of an inelastic, short-stretch compression bandage following total knee arthroplasty is a safe technique that is acceptable to patients. A larger, multicentre trial is required to determine its effect postoperatively.

Trial registration: The study was registered with Current Controlled Trials, identifier: ISRCTN86903140. Registered on 30 May 2013.

Keywords: Knee replacement, Arthroplasty, Compression bandage, Enhanced recovery, Fast track, Feasibility

\footnotetext{
*Correspondence: t.m.brock@doctors.org.uk

'Deceased

${ }^{1}$ Trauma and Orthopaedic Surgery, Newcastle University, Newcastle upon

Tyne NE1 7RU, UK

Full list of author information is available at the end of the article
} 


\section{Background}

Total knee arthroplasty is a common and highly successful operation in the management of osteoarthritis. However, postoperative knee swelling is a common problem due to intra-articular bleeding and inflammation of periarticular tissues [1]. This results in decreased functional performance as a result of quadriceps weakness [2] and arthrogenic reflex inhibition due to pain [3] which can delay rehabilitation, increase hospital length of stay and decrease patient-reported outcomes [4, 5]. Additionally, excessive knee swelling is associated with increased rates of wound dehiscence and infection [6].

Intraoperative techniques to reduce intra-articular bleeding, such as surgical technique [7], tourniquets [8] and medication [9] are features in enhanced recovery programmes. However, postoperative methods, including the use of a cold compress [10], cryotherapy [11], elastic bandaging [12] and compression bandages [13, 14] have had limited success.

Compression bandage therapy is the established treatment of venous ulcers and lymphoedema $[15,16]$. It is hypothesised that the application of this external compression aids venous return and reduces hydrostatic pressure in the leg by (1) improving the efficacy of the calf-muscle pump and (2) moving blood from the superficial to the deep venous system, subsequently allowing movement of fluid from the interstitial space. The use of inelastic bandages are preferred in arthroplasty as they have a low, tolerable resting pressure but a more effective activation of the deep venous system and calf-muscle pump with ambulation compared to their elastic counterparts [17].

The efficacy in total knee arthroplasty is still unclear due to conflicting results in the medical literature and heterogeneous methodology [13, 18-20]. We hypothesised that the use of a compression bandage in total knee replacement would improve postoperative pain, swelling and functional outcomes. The aim of this study was (1) to test study feasibility in the form of eligibility, recruitment rate, attrition rates and bandage complications, (2) to derive preliminary subjective and objective data and (3) to derive a power calculation for the Oxford Knee Score using preliminary data.

\section{Methods}

The study was a prospectively enrolled, randomised controlled feasibility study conducted at two hospital sites within Northumbria Healthcare NHS Foundation Trust. Ethical approval was obtained (13/NE/0137) and the study was registered with Current Controlled Trials (ISRCTN86903140). A protocol paper with complete methodology was published previously [21]. A Consolidated Standards of Reporting Trials (CONSORT) flow chart (Fig. 1) was utilised.

\section{Participant recruitment}

Fifty patients selected for total knee arthroplasty were enrolled in the study between November 2013 and June 2014. The inclusion criteria for the study included (1) primary total knee arthroplasty for osteoarthritis, (2) age over 18 years and (3) being able to provide written, informed consent. Exclusion criteria included (1) peripheral vascular disease characterised by an Ankle-Brachial Pressure Index (ABPI) <0.8, (2) peripheral neuropathy and (3) Body Mass Index (BMI) $>40$.

Patients were randomised on the day of surgery by electronic distance randomisation using the website http://www.sealedenvelope.com. The randomisation process was done in random permuted blocks to allow even balancing of the groups. Patients were allocated to the control group or the compression bandage group. There was no difference in baseline characteristics (Table 1). Preoperative measurements (below) were recorded prior to randomisation to reduce bias.

\section{Control group}

Patients underwent primary total knee arthroplasty under the care of one of two consultant orthopaedic surgeons (SM, MR). Surgery was performed under general anaesthesia or spinal anaesthesia and sedation. Intra-venously administered (IV) antibiotics (gentamicin $3 \mathrm{mg} / \mathrm{kg}$ and teicoplanin $400 \mathrm{mg} \mathrm{IV}$ ) and tranexamic acid (30 mg/ $\mathrm{kg}$ IV up to $2.5 \mathrm{~g}$ ) were administered and a tourniquet used. A Nexgen cruciate-retaining total knee arthroplasty was used (Zimmer, Swindon, United Kingdom) with Palacos $\mathrm{R}+\mathrm{G}$ bone cement (Heraeus Medical, Newbury, United Kingdom). Intra-operative periarticular injections of $80 \mathrm{ml} 0.125 \%$ bupivacaine were infiltrated and a further $20-\mathrm{ml} 0.125 \%$ bupivacaine bolus given via intra-articular wound catheter after wound closure. The skin was closed using surgical skin clips, which were removed at 10 to 14 days post-operatively. A hydrocolloid dressing (Aquacel Surgical, Convatec Ltd., Flintfield, UK) was used for the wound. Standard bandaging consists of a soft inner layer (Soffban, BSN Medical Ltd., Brierfield, UK) applied from $10 \mathrm{~cm}$ below to $10 \mathrm{~cm}$ above the patella with a $50 \%$ overlap of bandage, followed by a similar outer layer of crepe bandage (BSN Medical Ltd., Brierfield, UK) prior to deflation of the tourniquet.

The bandage and wound catheter were removed at $24 \mathrm{~h}$ leaving the hydrocolloid wound dressing in situ. This dressing stayed on until the clips were removed at 10-14 days. A cryocuff was used after $24 \mathrm{~h}$.

\section{Compression bandage group}

Patients received a compression bandage over the hydrocolloid surgical wound dressing instead of the routine wool and crepe bandage. Following tourniquet removal a soft inner layer (Soffban, BSN Medical Ltd., Brierfield, UK) was applied from the toes to the groin on the 


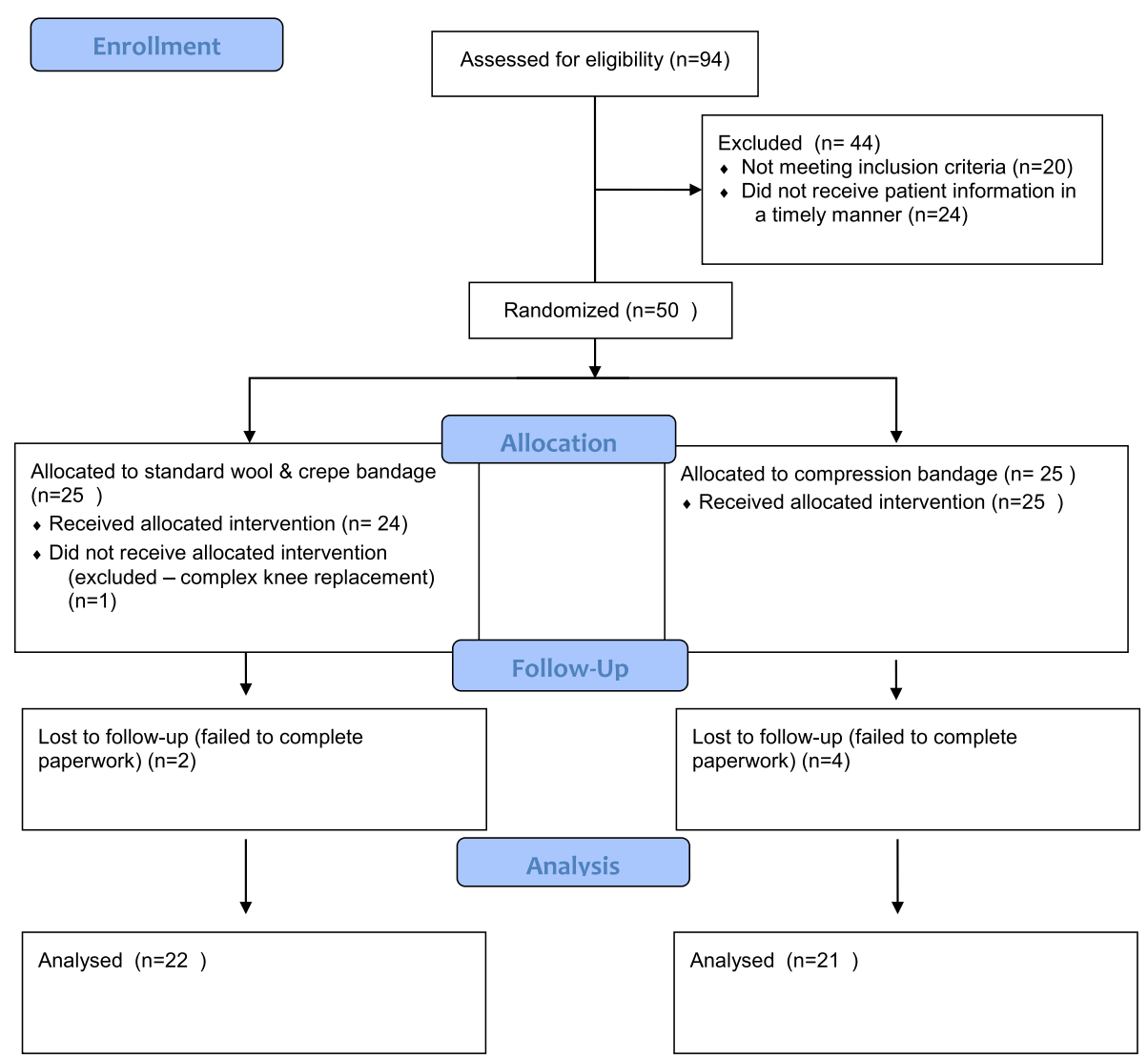

Fig. 1 Consolidated Standards of Reporting Trials (CONSORT) flow chart

affected leg with a $50 \%$ overlap of bandage. Following this the outer compressive layer bandage (Actico bandage, Activa Healthcare Ltd., UK) was applied firmly over the top, again with a $50 \%$ overlap of bandage. The bandage was pulled to full stretch before it was wrapped around the leg to ensure adequate compression in the application. It was applied after release of the tourniquet by necessity due to its length up the thigh. To ensure

Table 1 Patient demographics. Standard deviations in parentheses

\begin{tabular}{lll}
\hline & Control $(n=25)$ & Compression $(n=24)$ \\
\hline Age & $69.5(6.8)$ & $67.3(8.2)$ \\
Female gender & 16 & 16 \\
Body Mass Index & $28.8(4.4)$ & $29.7(5.5)$ \\
Oxford Knee Score & $23.3(7.9)$ & $22.7(8.3)$ \\
EQ-5D-3 L index score & $0.554(0.270)$ & $0.570(0.240)$ \\
Knee ROM (degrees) & $93.0(28.4)$ & $103.7(15.7)$ \\
Calf circumference (cm) & $37.3(4.3)$ & $37.8(3.2)$ \\
Knee girth (cm) & $41.7(4.1)$ & $41.8(3.9)$ \\
Thigh girth (cm) & $46.0(6.0)$ & $46.9(4.5)$ \\
Visual analogue pain score & $4.8(2.4)$ & $4.6(2.7)$ \\
\hline
\end{tabular}

ROM range of movement homogeneity in bandage application, the operating surgeons were shown a training video on correct application of the bandage and were given a tutorial on bandage application with real-life bandage application and feedback. The bandage was removed at $24 \mathrm{~h}$ post surgery leaving the hydrocolloid wound dressing in situ.

\section{Study feasibility}

Recruitment, retention and refusal rates were calculated. The proportion of patients undergoing primary total knee arthroplasty who did not meet the eligibility criteria was also calculated to ensure this was not too restrictive. Ease of application and tolerance of the compression bandage was determined qualitatively by the operating surgeons and patients, respectively. Potential adverse risks associated with the use of the compression bandage, such as pain, wound dehiscence and blistering, were recorded and rates calculated.

\section{Outcome measures}

The Oxford Knee Score was measured preoperatively and at 6 months in line with UK Health and Social Care Programme Patient Reported Outcome Measures. The 
EuroQol EQ-5D-3L questionnaire was measured preoperatively and at 6 months.

Knee swelling and range of motion were measured preoperatively, daily until discharge and at 6 weeks. Visual analogue pain scores were recorded preoperatively, daily until discharge (pre- and post-physiotherapy) and at 6 weeks.

Length of hospital stay, readmission rates and complications were also recorded using hospital episode statistic data.

\section{Statistical analysis}

A secure Excel database was used to record data (Microsoft Inc., Albuquerque, NM, USA). Statistical analysis was performed using SPSS version 23.0 (SPSS Inc., Chicago, IL, USA).

For each outcome measure (Oxford Knee Score, EQ$5 \mathrm{D}-3 \mathrm{~L}$ index score, knee swelling, range of motion, visual analogue scale score) the two groups were compared at each applicable set time point (preoperative, day 1 , day 2, 6 weeks, 6 months) using analysis of covariance (ANCOVA) with the baseline preoperative measurement set as the co-variate. Statistical significance was denoted at $p<0.05$.

A power calculation was performed based on the mean and standard deviations of the Oxford Knee Scores collected from this data to inform a future, larger trial. The risk of a type 1 error was set at 0.05 and a type II error at 0.20 . The retention rate from feasibility data was incorporated into the final sample size number to allow for possible patient dropout.

\section{Results}

\section{Study feasibility}

Of the 94 total knee arthroplasties performed during the time period, 20 (21\%) did not meet the inclusion criteria. Using the 74 eligible patients, the recruitment rate was $68 \%(n=50)$. Thirty-two percent $(n=24)$ of patients were seen in clinic but did not receive patient information in a timely manner and were not enrolled. There were no refusals of participation in the study.

During the trial, one patient was excluded from the trial in the control group due to an unplanned complex knee replacement which did not meet the inclusion criteria. Two patients in the control group and four patients in the compression bandage group did not complete 6-month follow-up and could not be analysed (dropout rate 12\%) (Fig. 1).

There were no reported problems with bandage application by the operating surgeons and the bandage was well-tolerated by patients, with no reports of discomfort or restriction. There were no reported skin complications with the compression bandages.

\section{Outcomes}

\section{Oxford Knee Score}

There was a mean Oxford Knee Score improvement of 11.0 in the control group and 13.1 in the compression bandage group. Whilst the point estimate (2.1) was nearing the minimally clinically significant difference of 3.0, the change in score was not statistically significant $(p=0.580 ; 95 \%$ CI -3.288 to 7.449 ) (Table 2$)$

2. EQ-5D-3L index

There was a mean improvement in the EQ-5D-3L index score in the compression bandage group compared to the control group at 6 months, but this was not statistically significant $(p=0.057$; point estimate $=0.147 ; 95 \% \mathrm{CI}-0.328$ to 0.005 ) (Table 2 )

3. Knee swelling

There was an increase in mean knee swelling in both groups on day 1 post-operatively, which increased further at day 2 post-operatively. However, these values were close to pre-operative levels by week 6 , with the exception of the knee circumference in both groups. There was, however, no significant statistical difference between groups at any time point (Fig. 2)

4. Range of motion

There was a decline in mean range of motion postoperatively in both groups, which decreased further at day 2 post-operatively. However, similarly to knee swelling, these values were close to pre-operative levels by week 6 . There was large variation in initial measurements in both groups, reflecting the varying degrees of restriction of the knee due to osteoarthritis and fixed-flexion deformity. There was no significant statistical difference between groups at any time point (Fig. 3)

\section{Pain scores}

There was an improvement between pain scores in both groups at 6 weeks compared to pre-operatively. There was a small increase in pain scores in the

Table 2 Oxford Knee Score and EQ-5D-3L index measurements - standard deviations in parentheses. Point estimate refers to mean difference between groups

\begin{tabular}{lllcll}
\hline & Control $(n=25)$ & Compression $(n=24)$ & Point estimate & $p$ value & $95 \%$ Cls \\
\hline Pre-op EQ-5D-3L index & $0.554(0.270)$ & $0.570(0.240)$ & 0.016 & - & - \\
6-month EQ-5D-3L index & $0.651(0.331)$ & $0.812(0.183)$ & 0.163 & 0.057 & -0.328 to 0.005 \\
Pre-op Oxford score & $23.3(7.9)$ & $22.7(8.3)$ & -0.6 & - & - \\
6-month Oxford score & $34.3(10.6)$ & $35.8(7.7)$ & 1.5 & 0.580 & -3.288 to 7.449 \\
\hline
\end{tabular}




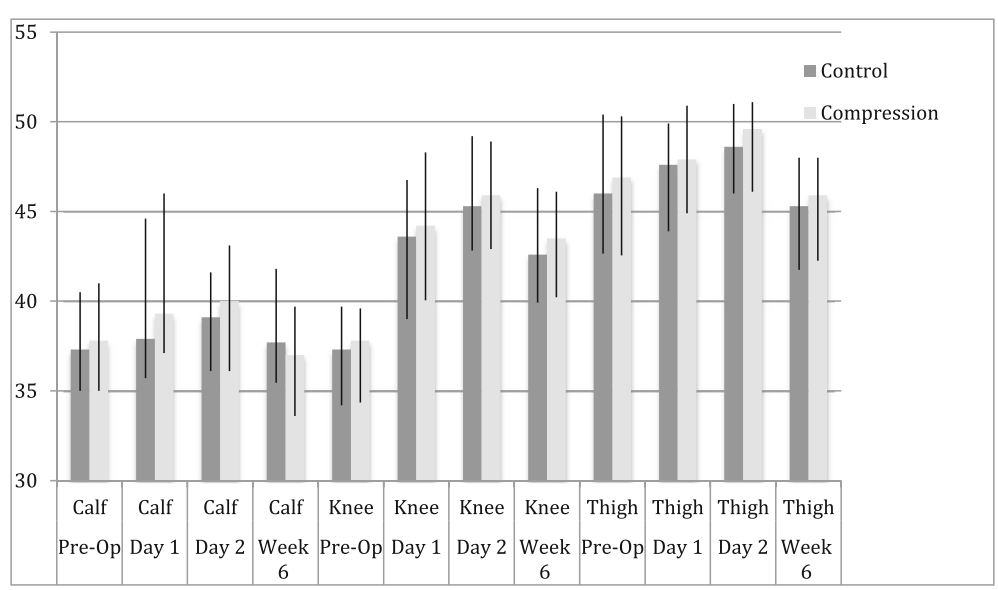

Fig. 2 Bar chart to show mean circumference $(\mathrm{cm})$ of the leg pre- and post-total knee arthroplasty. Error bars represent interquartile range. No statistical significance was found between groups at any time point

compression bandage group on day 1 and day 2 but this was not significant. There was no significant statistical difference in pain scores between groups at any time point (Table 3$)$

6. Length of stay and complications

The mean length of stay was 3.3 days in the control group and 3.1 days in the compression group $(p=$ 0.749 ; point estimate $=0.2 ; 95 \% \mathrm{CI}-0.815$ to 1.125 ). There were no registered infections or thromboembolic events in either group

\section{Discussion}

The main finding of this feasibility study was that the study was acceptable to patients with $68 \%$ recruitment rate of eligible patients. Attrition was relatively low at $12 \%$ and the intervention was acceptable to patients with no documented complications associated with the bandage use.
In terms of clinical outcomes there was a greater mean improvement in the Oxford Knee Score in the compression bandage group. Whilst the $95 \%$ confidence interval (-3.288 to 7.449) was wide it did include the three-point difference associated with a clinically important difference. There was a mean greater mean improvement in EQ-5D-3L index scores at 6 months in the compression bandage group, but this was not statistically significant $(p=0.057$; point estimate $=0.147 ; 95 \%$ CI -0.328 to 0.005). No statistical difference was found between the groups regarding knee range of motion, knee swelling or pain at any time point.

We accept that there are limitations with the current study. With regards to the study protocol, it was not possible to blind patients or investigators to the intervention, which may introduce bias. Secondly, interobserver variation is a known limitation of objective knee measurements including range of motion and



Fig. 3 Bar chart to show mean total range of motion (degrees) of the leg pre- and post-total knee arthroplasty. Error bars represent inter-quartile range. No statistical significance was found between groups at any time point 
Table 3 Mean visual analogue pain score - standard deviations in parentheses. Point estimate refers to mean difference between groups

\begin{tabular}{|c|c|c|c|c|c|}
\hline & Control $(n=25)$ & Compression $(n=24)$ & Point estimate & $p$ value & $95 \% \mathrm{Cls}$ \\
\hline Pre-op & $4.8(2.4)$ & $4.6(2.7)$ & -0.2 & - & - \\
\hline Day 1 pre physio & $3.9(2.7)$ & $4.5(2.7)$ & 0.6 & 0.974 & -1.660 to 1.685 \\
\hline Day 1 post physio & $5.1(2.6)$ & $6.0(2.6)$ & 0.9 & 0.307 & -0.802 to 2.376 \\
\hline Day 2 pre physio & $2.4(2.3)$ & $4.2(2.3)$ & 1.8 & 0.067 & -0.180 to 2.625 \\
\hline Day 2 post physio & $4.5(2.3)$ & $5.2(2.7)$ & 0.7 & 0.726 & -1.144 to 1.829 \\
\hline Week 6 & $2.6(1.6)$ & $2.8(2.2)$ & 0.2 & 0.244 & -0.560 to 2.110 \\
\hline
\end{tabular}

circumference. It is possible that this may account for some of the variation between subjects and consequently, in combination with the large sample size required for the definitive trial, will not be used. Thirdly, there was a large proportion of patients who were eligible for the trial but not enrolled (32\%). This was due to the logistics of research nurse availability and not patient demographics or comorbidities. All patients who were approached regarding the trial agreed to participate. Finally, whilst the primary aim was to ascertain feasibility of the study, the study measurements were underpowered and at risk of type II error. Using this preliminary data for Oxford Knee Score, incorporating attrition rate and standard deviation with $90 \%$ power, we would need the following number of participants to determine statistical significance: 354; 790; 3088 for a 3-, 2 - and 1-point difference, respectively. These figures will form the basis of the future multicentre study.

In a randomised controlled trial of 60 patients undergoing unicondylar knee arthroplasty, Pinsornsak et al. found no difference in swelling, blood loss or pain at $24 \mathrm{~h}$ and $48 \mathrm{~h}$ postoperatively between a modified Robert-Jones bandage and a standard wool and crepe bandage [19]. Whilst the bandaging technique was different, the findings are similar to our study.

In contrast, Charalambides et al. found that patients had improved range of motion (knee flexion) and decreased length of stay when a compression bandage was used [14]. However, the study of 150 patients was not randomised and relied on retrospective data in the cohort that received the standard wool and crepe bandages. It was also not performed in an enhanced recovery programme setting. A study by Cheung et al. found similar findings regarding improved knee flexion in the compression bandage group, with associated lower incidence of walking aids at discharge [20]. Anderson et al. found that the use of a compression bandage and local anaesthetic infiltration was associated with significant improvement in pain scores at $8 \mathrm{~h}$ but not $24 \mathrm{~h}$ postoperatively [13]. In our present study, no significant difference in pain scores were seen $24 \mathrm{~h}$ postoperatively. The local anaesthetic infiltration in both our groups may have influenced this. There was a small, non-significant increase in pain scores in the compression bandage group during day 1 and day 2 post-operatively, but this did not tally with qualitative information on the bandage provided by the patients.

To our knowledge this is the first study to utilise patient-reported outcome measures with the use of compression bandages after total knee arthroplasty. Whilst not statistically significant, the mean improvement in Oxford Knee Score of 2.1 in the compression bandage group is near the minimal clinically significant difference for the Oxford Knee Score [22]. The greater mean improvement in the EQ-5D-3L index score $(p=0.057$; point estimate $=$ $0.147 ; 95 \%$ CI -0.328 to 0.005 ) in the compression group also follows this trend. However, these results should be interpreted with caution due to the small sample size. As the compression bandage was easy to apply and well-tolerated by patients, without any documented complications, further investigation of its use in a larger, multicentre trial is warranted.

\section{Conclusion}

Feasibility data suggests that the compression bandage is easy to use and well-tolerated. No statistically significant differences were seen for post-operative scores between groups. However, there was a marginal trend to an improvement in patient-reported outcome measures at 6 months in the compression bandage group. Future work should be directed at further investigation of these patient-reported outcome measures in a larger, multicentre trial.

\section{Acknowledgements}

The authors are very grateful to York Trials Unit, UK for the definitive trial power analysis and manuscript advice. The authors would like to thank the participants involved in the study and the research team, Chris Herriot (clinical research nurse), Clare Casson and Diane Williams (senior physiotherapists).

\section{Funding}

The study was funded by Northumbria Healthcare NHS Foundation Trust.

Availability of data and materials

Data available on request.

\section{Authors' contributions}

This manuscript outlines independent investigator-initiated research. The views expressed are solely those of the authors. MR conceived the study, designed the study, participated in recruitment and reviewed the manuscript. TB designed the study, participated in recruitment and wrote the manuscript. AS 
designed the study. SM participated in recruitment and reviewed the manuscript. With the exception of AS, who is now deceased, all authors were involved in drafting the manuscript and revising it for important intellectual content and give permission for this version to be published.

\section{Authors' information}

TB is a specialist registrar in trauma and orthopaedic surgery. SM and MR are consultant orthopaedic surgeons.

\section{Competing interests}

The authors declare that they have no competing interests.

\section{Ethical approval and consent to participate}

Ethical approval was obtained (13/NE/0137) and the study was registered with Current Controlled Trials (ISRCTN86903140). Informed consent was given by all participants.

\section{Author details}

${ }^{1}$ Trauma and Orthopaedic Surgery, Newcastle University, Newcastle upon Tyne NE1 7RU, UK. ${ }^{2}$ Trauma and Orthopaedics, Warwick Clinical Trials Unit, University of Warwick, Coventry CV4 7AL, UK. ${ }^{3}$ Wansbeck General Hospital, Northumbria Healthcare NHS Foundation Trust, Woodhorn Lane, Ashington NE63 9J, UK.

Received: 17 February 2016 Accepted: 21 December 2016

Published online: 09 January 2017

\section{References}

1. Holm B, Kristensen MT, Bencke J, Husted H, Kehlet H, Bandholm T. Loss of knee-extension strength is related to knee swelling after total knee arthroplasty. Arch Phys Med Rehabil. 2010;91:1770-6.

2. Mizner RL, Snyder-Mackler L. Altered loading during walking and sit-tostand is affected by quadriceps weakness after total knee arthroplasty. J Orthop Res. 2005;23:1083-90

3. Fahrer H, Rentsch HU, Gerber NJ, Beyeler C, Hess CW, Grunig B. Knee effusion and reflex inhibition of the quadriceps. A bar to effective retraining. J Bone Joint Surg. 1988;70:635-8.

4. Moretti B, Notarnicola A, Moretti L, Setti S, De Terlizzi F, Pesce V, et al. I-ONE therapy in patients undergoing total knee arthroplasty: a prospective, randomized and controlled study. BMC Musculoskelet Disord. 2012;13:88.

5. Williams DP, O'Brien S, Doran E, Price AJ, Beard DJ, Murray DW, et al. Early postoperative predictors of satisfaction following total knee arthroplasty. Knee. 2013;20:442-6.

6. Yu GV, Schubert EK, Khoury WE. The Jones compression bandage. Review and clinical applications. J Am Podiatr Med Assoc. 2002;92:221-31.

7. Kumar N, Saleh J, Gardiner E, Devadoss VG, Howell FR. Plugging the intramedullary canal of the femur in total knee arthroplasty: reduction in postoperative blood loss. J Arthroplasty. 2000;15:947-9.

8. Levine BR, Haughom B, Strong B, Hellman M, Frank RM. Blood management strategies for total knee arthroplasty. J Am Acad Orthop Surg. 2014;22:361-71.

9. Martin JG, Cassatt KB, Kincaid-Cinnamon KA, Westendorf DS, Garton AS, Lemke $\mathrm{JH}$. Topical administration of tranexamic acid in primary total hip and total knee arthroplasty. J Arthroplasty. 2014;29(5):889-94.

10. Morsi E. Continuous-flow cold therapy after total knee arthroplasty. J Arthroplasty. 2002;17:718-22.

11. Adie S, Kwan A, Naylor JM, Harris IA, Mittal R. Cryotherapy following total knee replacement. Cochrane Database Syst Rev. 2012;9:CD007911.

12. Hughes DL, Crosby AC. Treatment of knee sprains: modified Robert Jones or elastic support bandage? J Accid Emerg Med. 1995:12:115-8.

13. Andersen LO, Husted H, Otte KS, Kristensen BB, Kehlet $\mathrm{H}$. A compression bandage improves local infiltration analgesia in total knee arthroplasty. Acta Orthop. 2008;79:806-11.

14. Charalambides C, Beer M, Melhuish J, Williams RJ, Cobb AG. Bandaging technique after knee replacement. Acta Orthop. 2005;76:89-94.

15. Franks PJ, Moody M, Moffatt CJ, Martin R, Blewett R, Seymour E, et al. Randomized trial of cohesive short-stretch versus four-layer bandaging in the management of venous ulceration. Wound Repair Regen. 2004;12:157-62.

16. Pike C. Using Actico bandaging for chronic oedema/lymphoedema management. Br J Nurs. 2011;20(1246):8-51.
17. Spence RK, Cahall E. Inelastic versus elastic leg compression in chronic venous insufficiency: a comparison of limb size and venous hemodynamics. J Vasc Surg. 1996;24:783-7.

18. Munk S, Jensen NJ, Andersen I, Kehlet $H_{1}$ Hansen TB. Effect of compression therapy on knee swelling and pain after total knee arthroplasty. Knee Surg Sports Traumatol Arthrosc. 2013;21:388-92.

19. Pinsornsak P, Chumchuen S. Can a modified Robert Jones bandage after knee arthroplasty reduce blood loss? A prospective randomized controlled trial. Clin Orthop Relat Res. 2013:471:1677-81.

20. Cheung A, Lykostratis H, Holloway I. Compression bandaging improves mobility following total knee replacement in an enhanced recovery setting. J Perioper Pract. 2014;24(4):84-6.

21. Brock TM, Sprowson AP, Muller S, Reed MR. Short-stretch inelastic compression bandage in knee swelling following total knee arthroplasty study (STICKS): study protocol for a randomised controlled feasibility study. Trials. 2015;16(1):87.

22. Murray DW, Fitzpatrick R, Rogers K, Pandit H, Beard DJ, Carr AJ, et al. The use of the Oxford Hip and Knee scores. J Bone Joint Surg. 2007;89-B(8):1010-4.

\section{Submit your next manuscript to BioMed Central and we will help you at every step:}

- We accept pre-submission inquiries

- Our selector tool helps you to find the most relevant journal

- We provide round the clock customer support

- Convenient online submission

- Thorough peer review

- Inclusion in PubMed and all major indexing services

- Maximum visibility for your research

Submit your manuscript at www.biomedcentral.com/submit 